\title{
Dysregulation of microRNA expression in diabetic skin
}

\author{
Enke Baldini $^{\mathrm{a}}$, Erika Testa ${ }^{\mathrm{b}}$, Christine Voellenkle $^{\mathrm{c}}$, Emanuela De Domenico $^{\mathrm{b}}$, \\ Francesca Cianfarani $^{\mathrm{b}}$, Fabio Martelli ${ }^{\mathrm{c}}$, Salvatore Ulisse ${ }^{\mathrm{a}}$, Teresa Odorisio ${ }^{\mathrm{b}, *}$ \\ a Dept. Surgical Sciences, Sapienza University of Rome, Italy \\ b Lab. Molecular and Cell Biology, Istituto Dermopatico dell'Immacolata, IDI-IRCCS, Via dei Monti di Creta, Rome, Italy \\ ${ }^{\mathrm{c}}$ Lab. Molecular Cardiology, IRCCS-Policlinico San Donato, San Donato Milanese, Italy
}

\section{A R T I C L E IN F O}

\section{Article history:}

Received 7 October 2019

Received in revised form 21 April 2020

Accepted 22 April 2020

\section{Keywords:}

miRNA biogenesis

Ulcer

pri-miRNA

Hypoxia

Oxidative stress

\begin{abstract}
A B S T R A C T
Background: Clinical skin manifestations are common in diabetes; however, molecular mechanisms underlying such defects are largely unknown. Several findings indicate a role for microRNAs (miRNAs) in skin homeostasis.

Objective: To investigate whether miRNA expression is altered in diabetic skin.

Methods: Type 1 and 2 mouse models of diabetes were used. MiRNA profiling was performed on RNA extracted from the skin of type 1 diabetic mice and non-diabetic controls. Expression levels of primiRNAs and of miRNA-biogenesis genes were also analyzed. Biogenesis gene expression analysis was performed in human dermal fibroblasts cultured in hyperglycemic, hypoxic or oxidative stress conditions.

Results: Several miRNAs were differentially expressed in diabetic skin with a general down-modulation as compared to controls. Bioinformatics analysis of signature-miRNA target genes showed the enrichment in pathways involved in skin homeostasis, such as TGF- $\beta$ and Wnt. MiRNA alteration in diabetic skin associated with reduced expression levels of DROSHA, DGCR8, XPO5, DICER1, AGO2, both as mRNA and protein. Reduced biogenesis gene expression did not correlate with accumulation of pri-miRNAs, which displayed differences in expression levels similar to those found for their mature miRNAs. Experiments with cultured fibroblasts showed that hypoxia and oxidative stress induced the down-regulation of miRNA-biogenesis genes in this skin cell type.

Conclusion: A general down-regulation of differentially expressed miRNAs was found in diabetic skin. This alteration is part of and is dependent from a wider transcriptional defect also affecting the expression of pri-miRNAs and of genes responsible for miRNA biogenesis. Such an alteration is likely contributing to diabetic skin manifestations.
\end{abstract}

(C) 2020 Japanese Society for Investigative Dermatology. Published by Elsevier B.V. All rights reserved.

\section{Introduction}

Diabetes is a chronic metabolic disease whose incidence and prevalence are high and constantly growing [1]. It arises as consequence of increased glucose levels due to insulin unbalance or impaired responsiveness. The vast majority of diabetic patients suffers from type 2 diabetes due to insulin resistance, while 5-10\% of patients are affected by type 1 insulin-dependent diabetes [1]. Clinical skin manifestations are common in diabetes, often being the first sign of the metabolic disorder [2]. They involve at least 30\% of patients and include increased propensity to infections, hyperpigmentation, dermal thickening, spontaneous blister development and the highly invalidating foot ulcers. Skin

\footnotetext{
* Corresponding author.

E-mail address: t.odorisio@idi.it (T. Odorisio).
}

complications are due to metabolic and physiological consequences typical of the diabetic condition. Impaired cell activity resulting from hyperglycemia, persistent inflammation and increased oxidative stress plays major roles in the healing defect in diabetic foot ulcer [3]. Glycation affects protein functions and strongly contributes to impaired cell activity in diabetes [4]. Altered gene expression also plays a role in diabetic dysfunctions; dysregulated gene expression was found in diabetic skin, with an increase in gene pathways involved in immune response and infectious diseases [5]. Moreover, differences in gene expression were associated with reduced biomechanical properties of diabetic skin [6]. A better understanding of the molecular alterations underlying skin defects in the diabetic condition may help in better managing diabetes-associated skin complications.

MicroRNA (miRNA) are small, non-coding RNAs regulating gene expression at post-transcriptional level. By binding to mRNA sequences in the $3^{\prime}$ untranslated region of target mRNAs, they 
primarily downregulate gene expression, thus playing a major role in all main biological processes, including skin development and homeostasis [7]. MiRNA dysregulation contributes to the onset and development of human disorders. As for diabetes, experimental findings indicate that miRNA altered expression is involved in diabetic complications, including the healing impairment of diabetic skin $[8,9]$.

To further investigate whether miRNA dysregulation plays a role in diabetic skin alterations, we compared miRNA baseline expression levels in diabetic and non-diabetic skin in a mouse model of type 1 diabetes by performing a wide miRNome analysis. Data were confirmed on type 2 diabetic mice. Having found miRNA widespread decrease in diabetic skin, the expression of genes involved in the miRNA biogenesis process as well as pri-miRNA levels were analyzed. The effect of hypoxia, hyperglycemia or oxidative stress in regulating miRNA-biogenesis genes in the skin context was also investigated in cultured dermal fibroblasts.

\section{Materials and methods}

\subsection{Diabetic mice}

Mice were purchased form Charles River Laboratories (Calco, Lecco, Italy). To obtain type 1 diabetic mice, six weeks-old C57Bl/6 male mice were intraperitoneally injected with $160 \mathrm{mg} / \mathrm{kg}$ streptozotocin (Sigma-Aldrich, St. Louis, MO) in $0.05 \mathrm{M}$ Na citrate $\mathrm{pH}$ 4.5. Seven days after, blood glucose levels were measured, and mice with glycemia above $250 \mathrm{mg} / \mathrm{dL}$ were included in the study. Three weeks later, glycemia was checked again, diabetic mice sacrificed and skin collected.

Ten weeks-old C57Bl/KS-Lepr $\mathrm{db} / \mathrm{db}$ male mice, homozygous for the spontaneous mutation of the leptin receptor (Lepr), and heterozygote littermates $(\mathrm{db} /+)$ were purchased. After one week, glycemia was checked, mice sacrificed and skin collected. $\mathrm{Db} / \mathrm{db}$ mice displayed glycemia above $500 \mathrm{mg} / \mathrm{dL}$; db/+ mice had a glycemia of $139 \pm 27.7 \mathrm{mg} / \mathrm{dL}$.

Animal procedures were approved by the Animal Committee of the Italian Ministry of Health (approvals n. SA-IDI-12-TO-1 and n. 1016/2016-PR).

\subsection{Mouse samples}

Back skin biopsies were snap-frozen or fixed in $10 \%$ formalin and paraffin-embedded. Frozen biopsies were crushed to powder that was poured into TRIzol (Termofisher Scientific, Waltham, MA, USA) for total RNA extractions. For protein extraction, the frozen powder was dissolved in RIPA buffer containing protease inhibitors; lysates were incubated in ice for $15 \mathrm{~min}$ and centrifuged at $16,000 \mathrm{~g}$ for $15 \mathrm{~min}$. Supernatants were frozen to $-80^{\circ} \mathrm{C}$.

Table 1

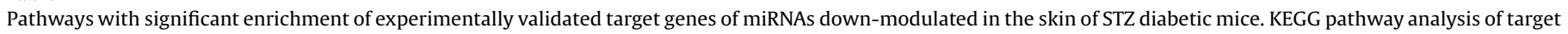

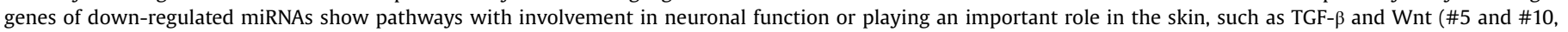
respectively). HIF-1 and VEGF signaling were also found among the significantly regulated pathways (\#29 and \#35, respectively).

\begin{tabular}{|c|c|c|c|c|}
\hline \# & KEGG pathway & n. genes & n. miRNAs & $p$-value \\
\hline 1 & Lysine degradation & 8 & 2 & $6.93 \times 10^{-11}$ \\
\hline 2 & Axon guidance & 16 & 2 & $3.31 \times 10^{-8}$ \\
\hline 3 & Gap junction & 8 & 3 & $5.72 \times 10^{-7}$ \\
\hline 4 & Retrograde endocannabinoid signaling & 12 & 2 & $1.30 \times 10^{-6}$ \\
\hline 5 & TGF- $\beta$ signaling & 9 & 4 & $3.49 \times 10^{-6}$ \\
\hline 6 & Long-term potentiation & 9 & 4 & $4.29 \times 10^{-6}$ \\
\hline 7 & Gastric acid secretion & 9 & 4 & $2.17 \times 10^{-5}$ \\
\hline 8 & Gabaergic synapse & 9 & 2 & 0.00017 \\
\hline 9 & GnRH signaling pathway & 9 & 4 & 0.00018 \\
\hline 10 & Wnt signaling pathway & 14 & 5 & 0.00021 \\
\hline 11 & Glutaminergic synapse & 11 & 2 & 0.00048 \\
\hline 12 & Dorso-ventral axis formation & 4 & 3 & 0.00048 \\
\hline 13 & ErbB signaling pathway & 9 & 5 & 0.00064 \\
\hline 14 & Dopaminergic synapse & 11 & 4 & 0.00130 \\
\hline 15 & Amphetamine addiction & 7 & 2 & 0.00133 \\
\hline 16 & Chagas disease & 9 & 2 & 0.00289 \\
\hline 17 & Circadian entrainment & 9 & 4 & 0.00330 \\
\hline 18 & Adherens junction & 8 & 2 & 0.00355 \\
\hline 19 & Cholinergic synapse & 9 & 4 & 0.00457 \\
\hline 20 & Nicotine addiction & 5 & 2 & 0.00565 \\
\hline 21 & Colorectal cancer & 6 & 3 & 0.00565 \\
\hline 22 & Long-term depression & 5 & 2 & 0.01398 \\
\hline 23 & Tubercolosis & 10 & 4 & 0.01631 \\
\hline 24 & Aldosterone-regulated sodium readsorption & 4 & 2 & 0.02006 \\
\hline 25 & Salivary secretion & 6 & 2 & 0.02006 \\
\hline 26 & Alzheimer's disease & 11 & 2 & 0.02006 \\
\hline 27 & Melanogenesis & 7 & 4 & 0.02006 \\
\hline 28 & Amiotrophic lateral sclerosis & 5 & 2 & 0.02024 \\
\hline 29 & HIF-1 signaling pathway & 8 & 2 & 0.02076 \\
\hline 30 & Pancreatic secretion & 6 & 2 & 0.02146 \\
\hline 31 & Salmonella infection & 6 & 2 & 0.02146 \\
\hline 32 & B cell receptor signaling pathway & 6 & 2 & 0.02305 \\
\hline 33 & Hepatitis B & 8 & 3 & 0.02683 \\
\hline 34 & Fatty acid elongation & 2 & 1 & 0.03625 \\
\hline 35 & VEGF signaling pathway & 5 & 2 & 0.03625 \\
\hline 36 & Serotoninergic synapse & 8 & 2 & 0.03625 \\
\hline 37 & NOD-like receptor signaling pathway & 5 & 1 & 0.03861 \\
\hline 38 & Morphine addiction & 7 & 2 & 0.03896 \\
\hline 39 & MAPK signaling pathway & 14 & 4 & 0.03896 \\
\hline 40 & Progesterone-mediated oocyte maturation & 6 & 2 & 0.03930 \\
\hline 41 & Spliceosome & 9 & 2 & 0.04113 \\
\hline
\end{tabular}




\subsection{MiRNA array}

Two-color hybridization was performed with miRCURY LNA microRNA arrays (7th generation [miRBase v18]; Exiqon, Vedbæk, Denmark), co-hybridizing total RNA from each type 1 diabetic mouse $(n=6)$ with total RNAs from a pool of 6 non-diabetic animals.

1.25 ug of total RNA was labeled with either Hy3 (non-diabetic pool) or Hy5 (diabetic) using the miRCURY LNA microRNA Array Power labeling kit (Exiqon), according to the manufacturer's protocol. Additionally, two dye-swaps were performed, labeling the diabetic sample with Hy3 and the control pool with Hy5.

Hybridization at $53{ }^{\circ} \mathrm{C}$ for $16 \mathrm{~h}$ and washing steps were carried out on the HS 400 PRO hybridization station (Tecan, Männedorf, Switzerland). MicroRNA arrays were scanned using the GenePix 4100A microarray scanner and the GenePix Pro GenePix Pro 6.1.0.4 software (Molecular Devices, San Jose, CA, USA).

\subsection{Global miRNA analysis}

Signal intensities were analyzed with the limma package (Bioconductor 2.5 on $\mathrm{R}$ 2.10.1) [10,11]. Background correction (normexp; cut-off $=10$ ) and within-array normalization (global LOESS) was performed. Differential expression and statistical significance were assessed for each miRNA species across all arrays using linear model fit and empirical Bayes method (lmFit, eBayes), taking into account the dye-swap. Spots showing intensities $<3$ SNR (for Hy3 and Hy5) OR displaying diameters $>130$ or $<70 \mu \mathrm{m}$ were considered as bad quality spots. The calling of a miRNA failed if out of its 4 replicates $\geq 2$ were bad quality spots and if this occurred on $\geq 4$ arrays. Normalized $\log 2$ ratios represent diabetic/ non-diabetic (Hy5/Hy3). P-values are adjusted for False Discovery Rate (Benjamini and Hochberg's method) [12]. Data are available on the NCBI Gene Expression Omnibus data base, GEO series GSE137605.

\subsection{MiRNA pathway analysis}

The list of differentially expressed miRNAs was filtered for significance using adjusted p-value of $\leq 0.10$ as threshold (Supplementary Table 1 ). The resulting list was separated by modulation, and down-modulated miRNAs were used for KEGGpathway analysis by DIANA miRPath v.2.0, selecting the experimentally validated miRNA interactions comprised in DIANATarBase [13].

\subsection{Real time $R T-P C R$}

Ten ng of total RNA were converted into cDNA with the TaqMan MicroRNA Reverse Transcription Kit (Applied Biosystems, Foster City, CA, USA), and templates were amplified in real time RT-PCR by using Premix Ex Taq (Probe qPCR) (Takara Bio, Shiga, Japan) and specific TaqMan MicroRNA Assays (Applied Biosystems). Otherwise, $5 \mu \mathrm{g}$ of RNA were retrotranscribed with M-MLV reverse transcriptase. The cDNAs were amplified with Premix Ex Taq (Probe qPCR) (Takara Bio) and specific TaqMan Pri-miRNA Assays (Applied Biosystems), or with the SYBR Premix Ex Taq II (Takara Bio) and primers for mRNA sequences (Supplementary Table 2). TATA-box binding protein $(T B P)$ was identified as most suitable reference gene for data normalization of both mRNAs and primiRNAs after having tested its mRNA levels and those of other five housekeeping genes in diabetic and non-diabetic samples. Candidate reference genes were ranked according to their expression stability by the geNorm application for Microsoft Excel (data not shown) [14]. MiR-16 displayed a similar expression in diabetic and non-diabetic samples ( $\log F C-0.16$, adj $p$-value 0.86), and was selected for miRNA normalization.

For PCR detected with the SYBR-Green dye, the relative expression ratio of target genes was computed with the LightCycler 2.0 Relative Quantification software (Roche Diagnostics, Basel, Switzerland), by applying the correction for PCR efficiency. For PCR performed with TaqMan probes, calculation of data was executed by means of the $\Delta \Delta C T$ method.

\subsection{Western blot}

Fifty $\mu \mathrm{g}$ of tissue extracts were electrophoresed on polyacrylamide gels and transferred onto nitrocellulose membranes. Membranes were saturated with $5 \%$ low fat milk in TBST (50 $\mathrm{mM}$ Tris- $\mathrm{HCl}, 150 \mathrm{mM} \mathrm{NaCl}$ and $0.05 \%$ Tween-20, $\mathrm{pH} 7.5$ ) for $2 \mathrm{~h}$ at room temperature. Incubations with primary antibodies were performed in TBST with 2.5\% low fat milk at $4{ }^{\circ} \mathrm{C}$ overnight. Antibodies were diluted as follows: Anti-XPO5 (ab131281, Abcam, Cambridge, UK) 1:3,000; anti-DGCR8 (ab191875, Abcam) 1:3000; anti-AGO2 (ab57113, Abcam) 1:1000; anti-DROSHA (ab12286, Abcam) 1:1000; anti-DICER1 (sc-30226, Santa Cruz Biotechnology, Dallas, TX, USA) 1:1000; anti- $\beta$-tubulin (T2200, Sigma-Aldrich) 1:20,000; anti-vinculin (ab129002, Abcam) 1:20,000. Horseradish peroxidase-conjugated secondary antibodies (Jackson ImmunoResearch, Cambridgeshire, UK) were used in TBST with $2.5 \%$ low fat milk for $1 \mathrm{~h}$. Western blots were revealed by chemiluminescence.

\subsection{Immunofluorescence}

Four-micrometer-sections of paraffin-embedded skin specimens were rehydrated and antigen retrieved prior staining with the anti-DICER1 antibody (sc-30226, Santa Cruz Biotechnology) diluted 1:25. Sections were analyzed with Axioplan2 fluorescence microscope (Carl Zeiss, Oberkochen, Germany) equipped with an Axiocam camera. Staining was quantified with ImageJ (imagej.nih. gov/ij/index.html) after applying a constant threshold.

\subsection{Culture conditions}

Human dermal primary fibroblasts isolated from foreskin samples were cultured in F10 medium (Gibco, Invitrogen, Carlsbad, CA, USA) containing $10 \%$ fetal bovine serum (FBS), $0.5 \%$ penicillin and $2 \%$ glutamine.

For hypoxic conditions, fibroblasts were maintained for $24 \mathrm{~h}$ (for RNA analysis) or $48 \mathrm{~h}$ (for protein analysis) in $1 \% \mathrm{O}_{2}$ by using a Hypoxia Incubator Chamber (Stemcell Technologies, Grenoble, France).

The oxidative stress was produced by adding hydrogen peroxide $\left(\mathrm{H}_{2} \mathrm{O}_{2}\right)$ to cultured fibroblasts at different doses $(0-200 \mu \mathrm{M})$ for the indicated times. Cell proliferation was measured by colorimetric immunoassay based on BrdU incorporation (Roche Diagnostics). Total cellular glutathione was detected after $4 \mathrm{~h}$ incubation by means of the GSH + GSSG/GSH Assay Kit (Abcam).

Chronic hyperglycemic conditions were obtained by culturing fibroblasts for three weeks in commercially available Dulbecco's Modified Eagle's Medium (DMEM, Sigma-Aldrich) containing 25 $\mathrm{mM}$ or $5.5 \mathrm{mM}$ D-glucose. The high glucose and low glucose DMEM had similar osmolarity.

\subsection{Statistical analysis}

The test U of Mann-Whitney was used to compare pri-miRNAs, miRNAs, mRNAs and protein levels in control and diabetic mice, while $t$-test was used for the in vitro experiments with cultured fibroblasts. 


\section{Results}

3.1. MiRNA profiling reveals a miRNA general down-modulation in the skin of diabetic mice

MiRNA profiling was performed by miRNome analysis on RNA extracted from the skin of streptozotocin-induced diabetic mice, a model of type 1 diabetes, and non-diabetic mice ( $n=6 /$ group). More than 400 different miRNA species were identified (GSE137605). Of these, 30 were significantly modulated, showing an FDR adjusted p-value $\leq 0.10$. Strikingly, among these 30,27 were down-regulated and only 3 were up-regulated in diabetic mice (Fig. 1 A, Supplementary Table 1). These findings were validated by real-time RT-PCR in skin samples from a different group of diabetic mice and non-diabetic controls ( $n=8 /$ group) by analyzing a subset of miRNAs (Fig. 1B). MiRNAs selected for validation were those found to be more down-regulated or having validated target genes (miR-541-5p, miR-136-5p, and miR-148a-3p). MiR-139-5p, one of the up-regulated miRNAs, was also analyzed for validation. All miRNAs examined were confirmed to be expressed at significantly different levels. The same miRNAs were analyzed in the skin of the $\mathrm{db} / \mathrm{db}$ mice, a model of type 2 diabetes, compared to $\mathrm{db} /+$ nondiabetic mice ( $n=6 /$ group). Differences in expression levels for selected miRNAs were found also in type 2 diabetic mice (Fig. $1 \mathrm{C}$ ).

KEGG pathway analysis on down-regulated miRNAs with validated targets was performed (Table1). Among the most involved pathways there were TGF- $\beta$ and Wnt signaling, both with proved roles in skin homeostasis, in wound healing and in diabetic complications [15-17]. Moreover, a number of target genes were involved in neuronal development and neurotransmission pathways. Hypoxia inducible factor- $1 \alpha$ (HIF- $1 \alpha$ ) and vascular endothelial growth factor (VEGF) signaling pathways, important for skin angiogenesis and in cutaneous wound healing [18-21], were also among the pathways significantly involved.

\subsection{Genes responsible for miRNA biogenesis are down-modulated in the skin of diabetic mice}

The higher number of down-modulated miRNAs in the skin of diabetic mice may reflect either a reduced transcription rate or an impairment of miRNA biogenesis in the diabetic condition. We therefore investigated whether the expression of genes involved in the canonical miRNA biogenesis pathway was altered in the diabetic skin. The following genes were analyzed: the ribonuclease III DROSHA and Di George Syndrome Critical Region 8 (DCGR8), both mediating primary miRNA (pri-miR) to precursor-miRNA (pre-miR) maturation within the nucleus; Exportin 5 (XPO5), allowing pre-miR exportation to the cytoplasm; the ribonuclease III DICER1 and the Argonaute member 2 (AGO2) of the miRNAinduced silencing complex (miRSC), responsible for miRNA terminal maturation in the cytoplasm [22]. All genes displayed significantly lower mRNA levels in the skin of streptozotocininduced diabetic mice $(n=8)$ and $\mathrm{db} / \mathrm{db}$ mice $(n=6)$ as compared to non-diabetic controls (Fig. 2A and B).

A

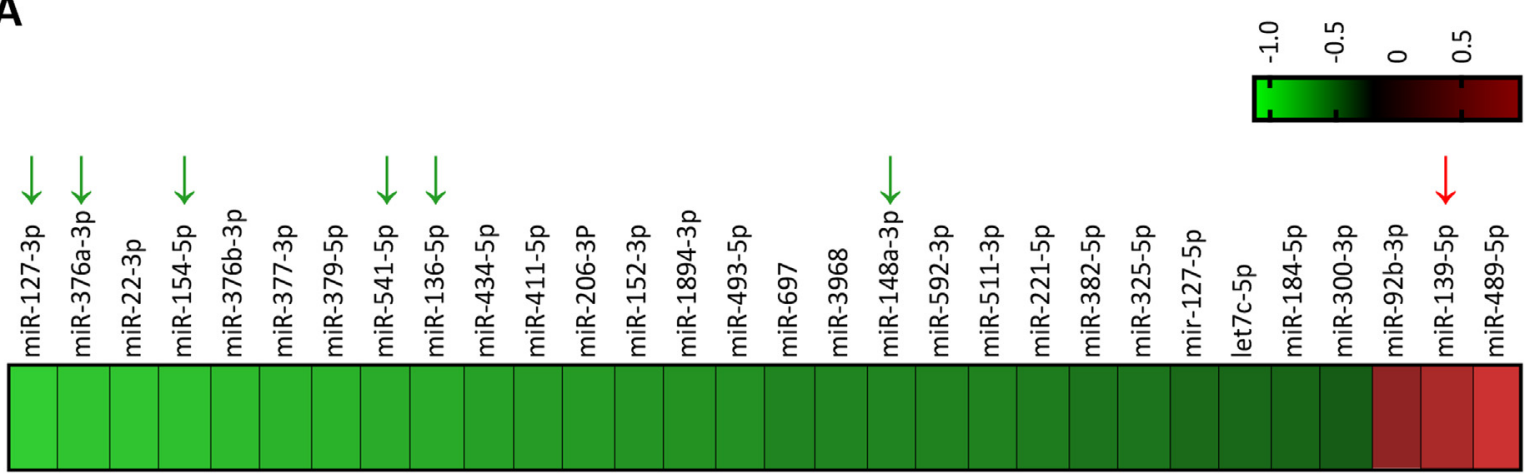

B

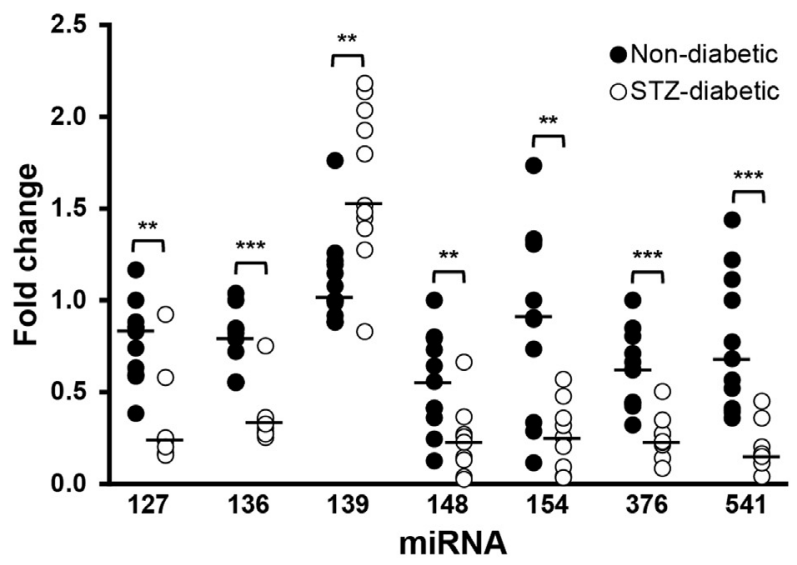

C

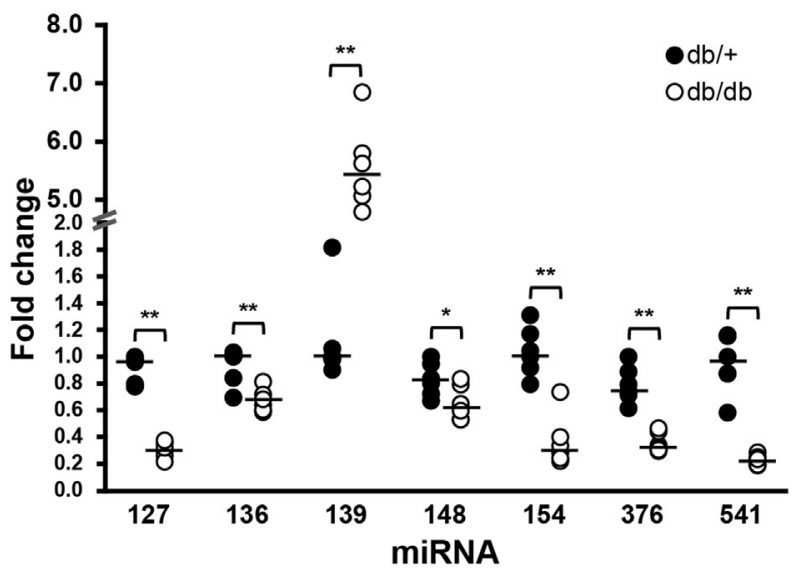

Fig. 1. Comparison of miRNA expression in the skin of diabetic and non-diabetic mice.

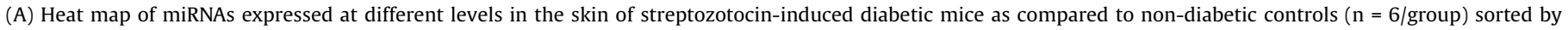

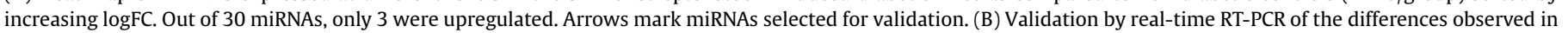

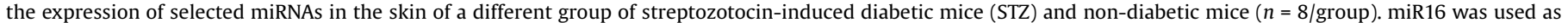

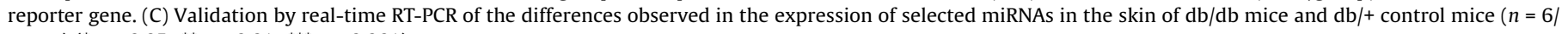
group) $\left({ }^{*} p<0.05 ;{ }^{* *} p<0.01 ;{ }^{* * *} p<0.001\right)$. 
A

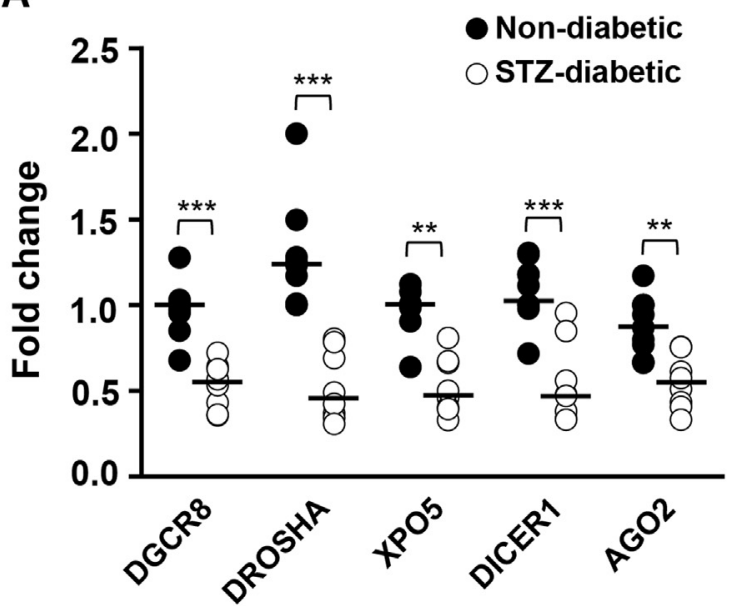

C

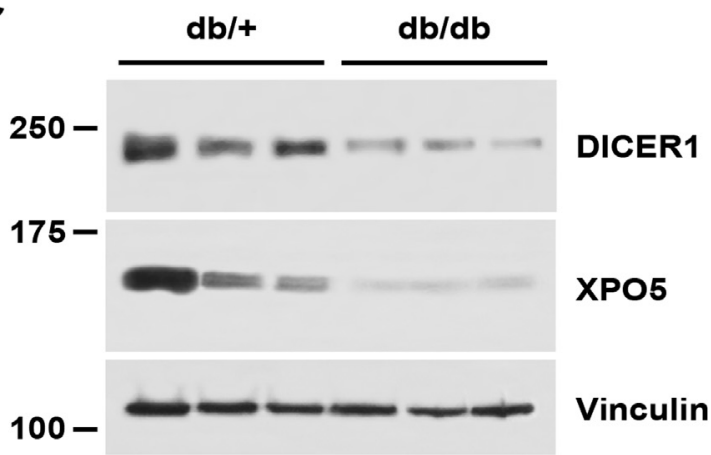

$\mathbf{E}$

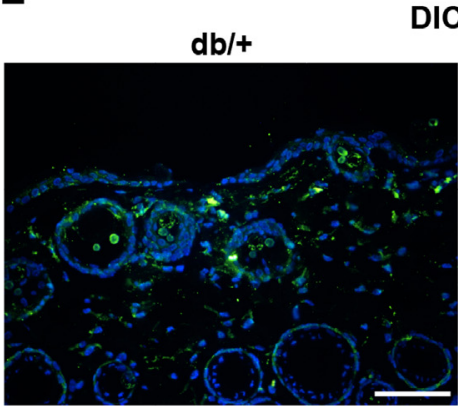

DICER1

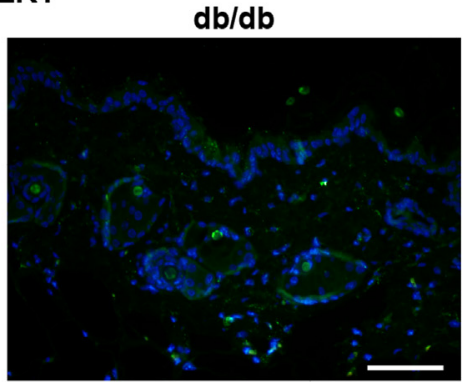

B

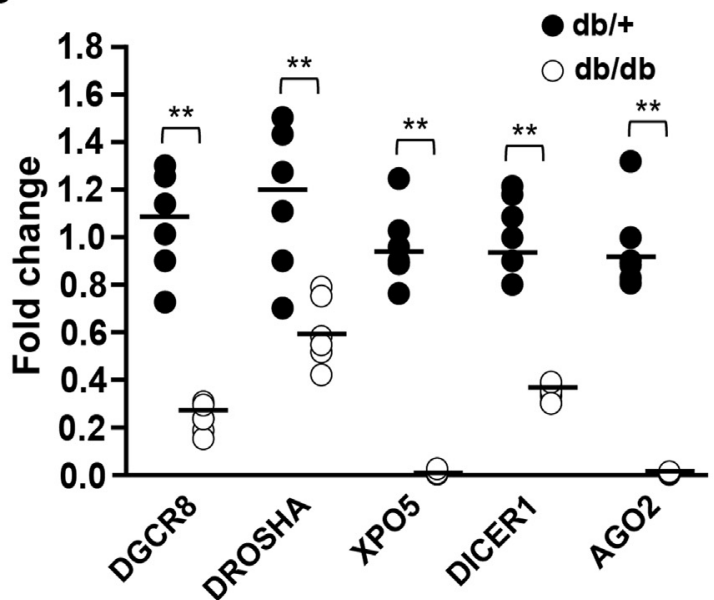

D

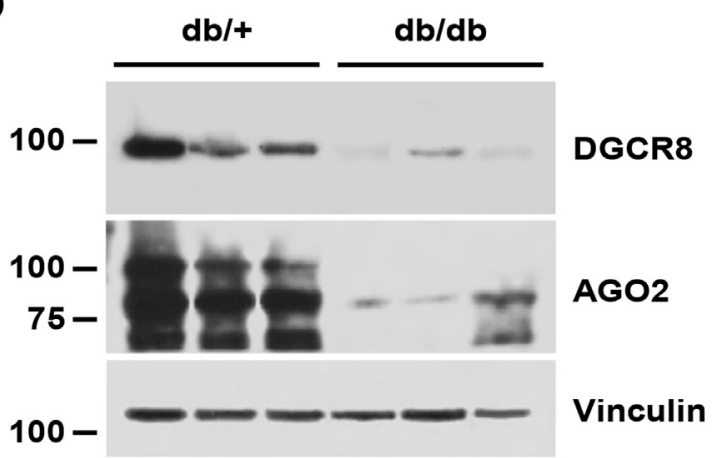

$\mathbf{F}$

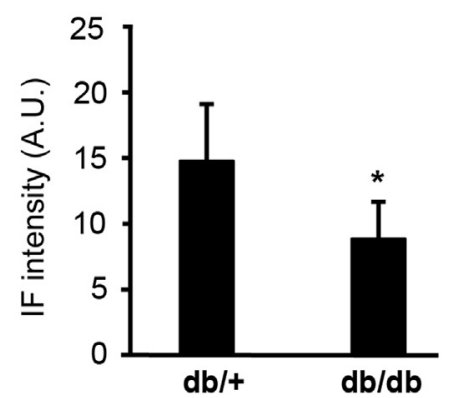

Fig. 2. Reduced expression levels of genes involved in miRNA biogenesis in the skin of diabetic mice.

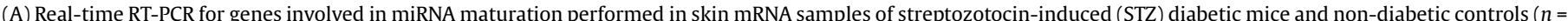

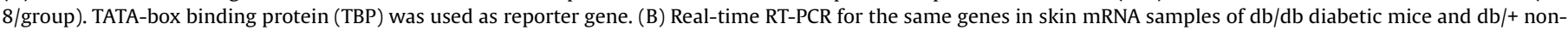

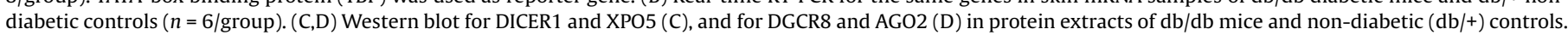

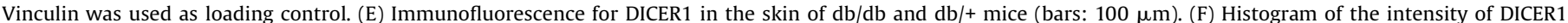
immunofluorescence staining in $\mathrm{db} / \mathrm{db}$ and $\mathrm{db} /+$ skin specimens $\left({ }^{*} p<0.05 ;{ }^{* *} p<0.01 ;{ }^{* * *} p<0.001\right)$.

Western blot on skin extracts confirmed a reduction of DGCR8, $\mathrm{XPO}$, DICER1 and AGO2 in diabetic mice as compared to nondiabetic controls (Fig. $2 \mathrm{C}$ and D). DROSHA antibodies did not detect any specific signal in skin samples of both diabetic and nondiabetic mice in our experimental conditions. DICER1 expression was further analyzed by immunofluorescence on skin sections of $\mathrm{db} / \mathrm{db}$ and $\mathrm{db} /+$ mice and indicated a general reduction in staining intensity in the skin of diabetic mice (Fig. $2 \mathrm{E}$ and F).

\subsection{Pri-miRNA expression levels in diabetic and non-diabetic skin reflect differences found in miRNA expression}

Having found a significant decrease in the expression levels of genes responsible for miRNA biogenesis in diabetic skin samples, we hypothesized that non-processed miRNA precursors accumulate in diabetic skin cells as compared to non-diabetic ones. Quantification of pri-miRNAs for the same miRNAs analyzed for miRnome validation was carried out by real-time RT-PCR (Fig. 3A and B). Contrary to our hypothesis, differences in pri-miRNA expression levels quite closely correlated with those found in mature miRNAs, with the only exception of pri-miR148 that was expressed at similar levels in $\mathrm{db} / \mathrm{db}$ mice and $\mathrm{db} /+$ controls.

3.4. Hypoxia and oxidative stress conditions down-modulate miRNA biogenesis genes in dermal fibroblasts

Diabetic skin microenvironment is subjected to reduced oxygen supply due to micro- and macro-angiopathy, and hypoxia plays an 
A

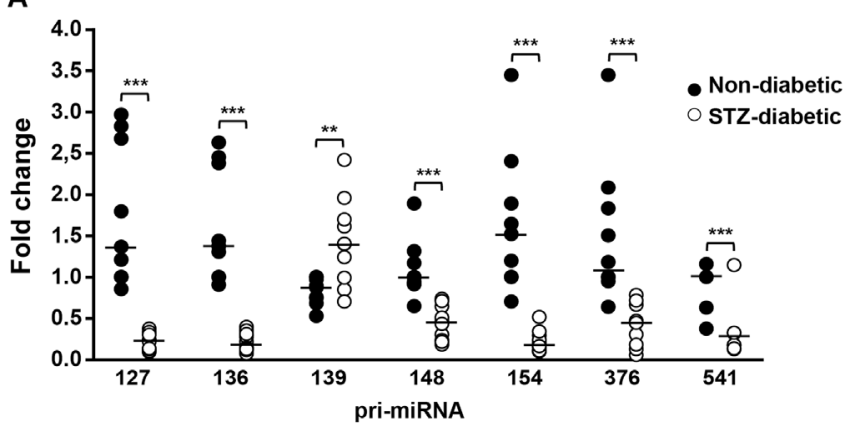

B

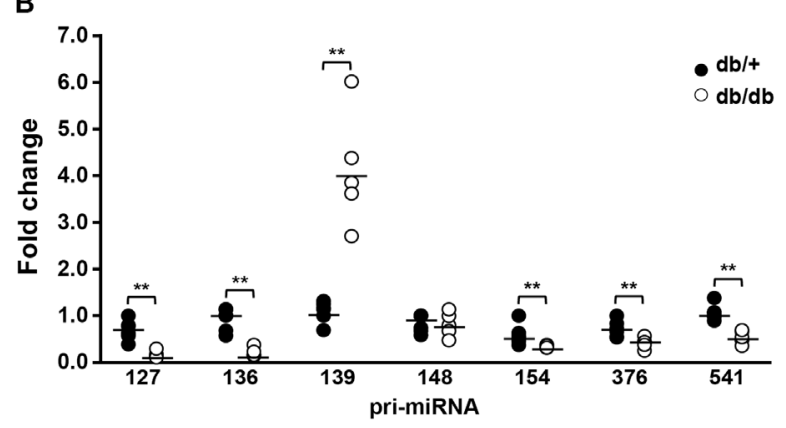

Fig. 3. Pri-miRNAs are also generally downregulated in the skin of diabetic mice. Real-time RT-PCR for pri-miRNAs of miRNAs tested for miRnome validation in skin RNA samples from streptozotocin-induced (STZ) diabetic mice and non-diabetic controls (A), or in $\mathrm{db} / \mathrm{db}$ and non-diabetic controls $(\mathrm{db} /+)(B)$. TBP was used as reporter gene $\left.{ }^{* *} p<0.01 ;{ }^{* * *} p<0.001\right)$.

important role in the development of diabetic complications [23]. The skin of animal models used in this study was shown to have reduced vascularization due to impaired angiogenic growth factor expression and altered endothelium responses [24,25].

A role for hypoxia in down-modulating DICER1 and DROSHA expression was reported in tumor contexts [26,27]. Therefore, we investigated whether the hypoxic condition could down-regulate the expression of miRNA-biogenesis genes also in the skin context. To this end, fibroblasts isolated from the skin of healthy donors were cultured in low oxygen levels $\left(1 \% \mathrm{O}_{2}\right)$ for 24 and $48 \mathrm{~h}$. To verify whether culture conditions were able to induce a response to hypoxia, the expression of the transcription factor HIF-1 $\alpha$ was analyzed. In cells exposed to low $\mathrm{O}_{2}$ for $48 \mathrm{~h}$, HIF- $1 \alpha$ protein levels were strongly increased in all fibroblast strains (Fig. 4A).

MRNA levels of DGCR8, DROSHA, and XPO5 were significantly reduced after $24 \mathrm{~h}$ of culture in hypoxic condition (Fig. $4 \mathrm{~B}$ ). Proteins levels for the same genes were also found to be significantly reduced in extracts of fibroblasts cultured for $48 \mathrm{~h}$ under hypoxia, as compared to those maintained in normoxic conditions (Fig. 4 C, D).

To test the effect of hyperglycemia on the expression of miRNA biogenesis genes, fibroblasts were cultured in $25 \mathrm{mM}$ glucose for three weeks, a condition mimicking chronic hyperglycemia [28]. Fibroblasts cultured in high glucose did not display modulation of any of the genes analyzed, both at mRNA (Fig. $4 \mathrm{E}$ ) and protein level (data not shown), as compared to those cultured in normoglycemic conditions.

As diabetes induces oxidative stress in the skin, the effect of oxidative stress on the expression levels of miRNA biogenesis genes was investigated by culturing fibroblasts in the presence of $\mathrm{H}_{2} \mathrm{O}_{2}$. At first, cellular proliferation was evaluated by treating cells with increasing $\mathrm{H}_{2} \mathrm{O}_{2}$ amounts for $48 \mathrm{~h}$ (Fig. 5A). The minimal dose capable of significantly impairing cell growth, a feature of oxidative-stress induced cell senescence, was $200 \mu \mathrm{M} \mathrm{H}_{2} \mathrm{O}_{2}$. The increase of total glutathione, a major cellular antioxidant, was detected after $4 \mathrm{~h}$ of incubation with $200 \mu \mathrm{M} \mathrm{H}_{2} \mathrm{O}_{2}$ confirming the alteration of the redox status of cells (Fig. 5B). Therefore, this concentration was used to test the effect of oxidative stress on biogenesis genes. Real time RT-PCR performed in cells cultured for $24 \mathrm{~h}$ in the presence of $\mathrm{H}_{2} \mathrm{O}_{2}$ revealed a significant reduction in mRNAs only for DGCR8 (Fig. 5C). Decreased expression was confirmed for DGCR8 and observed for XPO5 at protein level after $48 \mathrm{~h}$ of $\mathrm{H}_{2} \mathrm{O}_{2}$ treatment (Fig. 5D, E).

\section{Discussion}

Hyperglycemic exposure leads to profound gene expression alterations that persist after reversal to a normoglycemic condition [29]. Such a hyperglycemic memory plays a central role in the recurrence of diabetic complications and it depends on epigenetic changes related to activity of advanced glycation end products (AGE), enhanced reactive oxygen species (ROS) and inflammatory mediators [30]. MiRNAs contribute to the control of tissue homeostasis by mediating post-transcriptional regulation of gene expression, and they were found to be involved in the development of diabetic skin complications [7].

Herein, we investigated miRNA expression in the skin by using experimental mouse models of type 1 and type 2 diabetes. The skin of this animals shows features which resemble the alteration observed in human diabetic skin with regard to epidermal differentiation, dermal composition, increased inflammation, reduced vascularization, neuropathy and delayed wound healing [24,25,31-34].

In the streptozotocin-induced diabetic mice, we found differences in miRNA expression between diabetic and non-diabetic skin. Differences were validated in a subset of miRNAs in the same strain of mice and in the type 2 diabetic mouse model. Among these miRNAs, some have proved target genes involved in pathways with important roles in regulating skin homeostasis and in promoting diabetic skin complications, such as Wnt, TGF- $\beta$, HIF- $1 \alpha$, and VEGF-A signaling. HIF- $1 \alpha$ and VEGF-A are central players in the angiogenic response that it is impaired in diabetic skin and that is a major cause of diabetic ulcer formation [35]. In the diabetic environment, HIF- $1 \alpha$ stabilization following hypoxia is impaired due to hyperglycemia [28,36], with reduction in expression levels for HIF-1 target genes. Among those genes there are VEGF-A and the chemokine stroma cell derived factor-1 (SDF1 ), major players in the angiogenic response via endothelial cell activation and recruitment of endothelial progenitor cells [37]. On the other hand, in diabetic patients with ischemic cardiomyopathy, increased oxidative stress and signaling alterations induce a pseudo-hypoxia condition with mild chronic HIF-1 $\alpha$ activation [38]. Nevertheless, this constitutively active HIF-1 $\alpha$ is no longer sensitive to acute hypoxia.

Wnt and TGF- $\beta$ pathways are also involved in skin wound healing and play a role in tissue remodeling and extracellular matrix deposition [39-41], other aspects altered in diabetic skin. Moreover, Wnt is directly involved in insulin homeostasis, with opposing roles ascribed to canonical and non-canonical Wnt signaling [42].

Different gene pathways found to be regulated by differentially expressed miRNAs are involved in neuronal function; this is not surprising, as peripheral neuropathy is a major defect of diabetic skin, with important consequences for foot ulcer formation [43].

Interestingly, a global miRNA down-modulation was observed in diabetic compared to non-diabetic skin, suggesting that the diabetic skin microenvironment affects miRNA biogenesis. miRNA biogenesis is a multistep process involving several molecular mediators. Most miRNAs are processed via the canonical biogenesis pathway. Non-canonical pathways involve the same proteins of the canonical one, in combination with other proteins, 
A

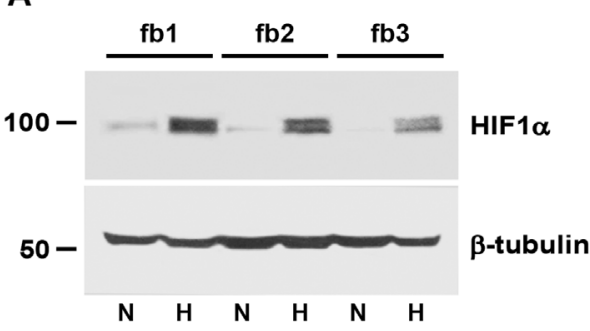

C

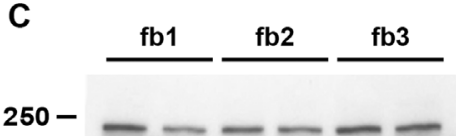

$250-$

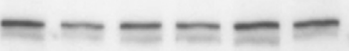

DICER1

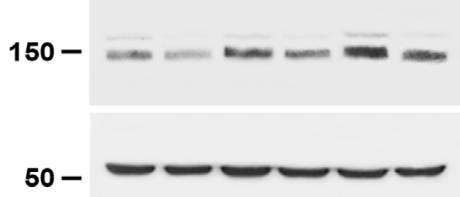

DROSHA

$\beta$-tubulin

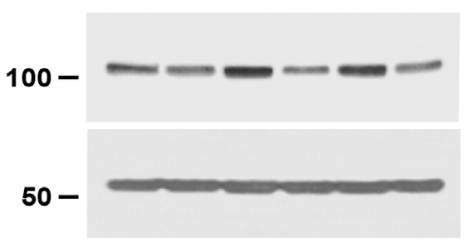

DGCR8

$\beta$-tubulin

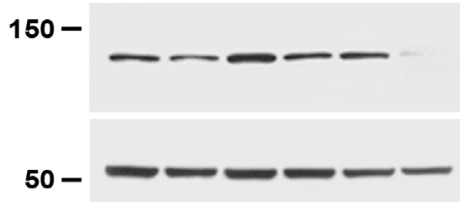

XPO5

$100-$

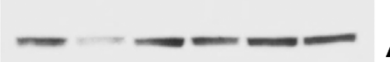

$50-$

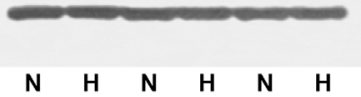

B

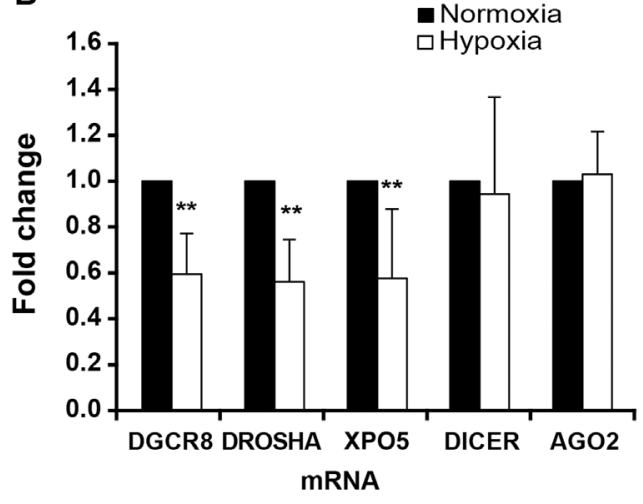

D

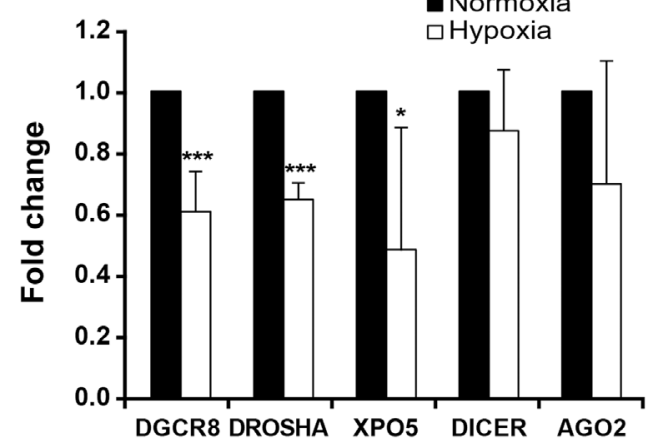

E

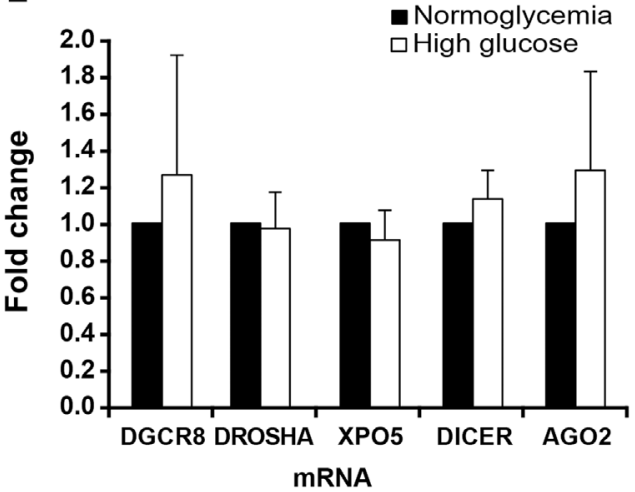

Fig. 4. Hypoxia down-modulates the expression levels of miRNA biogenesis genes in dermal fibroblasts.

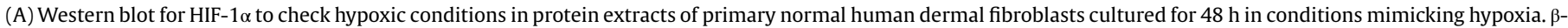

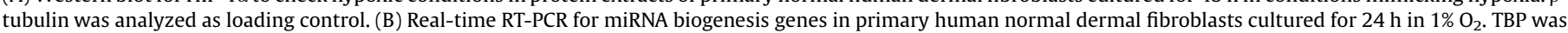

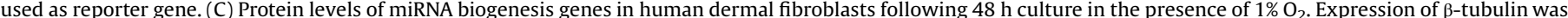

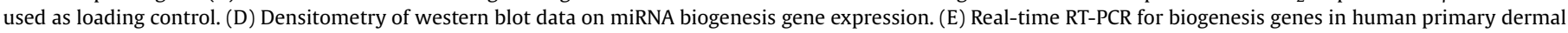
fibroblasts cultured in the presence of high glucose levels. TBP was used as reporter gene. $\mathrm{N}=$ normoxia; $\mathrm{H}=$ hypoxia $\left({ }^{*} p<0.05\right.$; $\left.{ }^{* *} p<0.01 ;{ }^{* * *} p<0.001\right)$.

but not all biogenesis proteins are involved in a single noncanonical pathway. We analyzed a subset of genes involved in the different steps of the canonical pathway: DROSHA, DGCR8, XPO5, DICER1 and AGO2. All of them were expressed at significantly reduced levels in the skin of both streptozotocin-dependent and $\mathrm{db} / \mathrm{db}$ diabetic mice, suggesting that reduced biogenesis gene levels account for altered miRNA expression in diabetic skin. Despite the observed reduced expression of biogenesis proteins, a minority of miRNAs were up-regulated in diabetic skin. Processing by non-canonical biogenesis pathways cannot explain the increased expression of these miRNAs, as all biogenesis genes analyzed were found to be down-regulated in diabetic skin. A hypothetic explanation is that the higher levels of a minority of miRNAs can be due to a transcriptional increase that overcomes the decrease in the efficiency of miRNA precursor processing.
MiRNAs found to be significantly down-regulated do not correspond to those that were reported to play a role in diabetic complications [8]. Our analysis, therefore, highlights novel miRNAs with potential role in skin homeostasis to be further investigated.

In cancer cells, deregulated miRNA biogenesis was correlated with hypoxic condition within the tumor and with pri-miR accumulation in cell nuclei [26,27]. Dermal human fibroblasts cultured in hypoxic conditions expressed reduced levels of DGCR8, DROSHA and XPO5, indicating that hypoxia down-regulates miRNA biogenesis genes in skin cells. The hypoxic conditions used in our experimental setting resemble severe acute ischemia found in chronic wounds. This data suggests that miRNA biogenesis gene down-modulation may have implications in the hypoxic environment of diabetic foot ulcers. Dermal fibroblasts cultured in the presence of $\mathrm{H}_{2} \mathrm{O}_{2}$ express reduced levels of DGCR8 
A

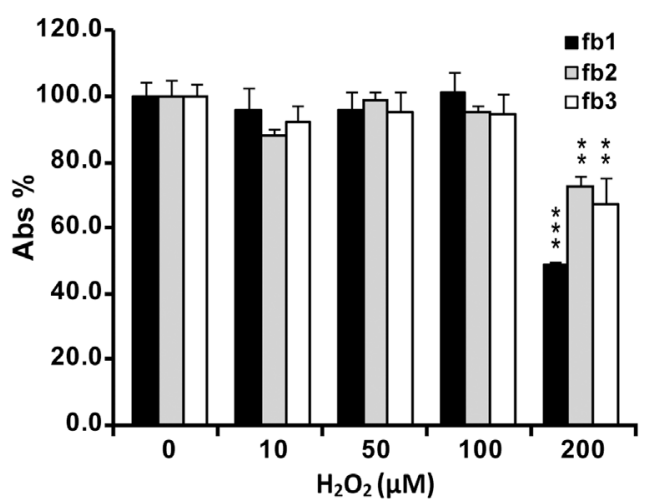

C

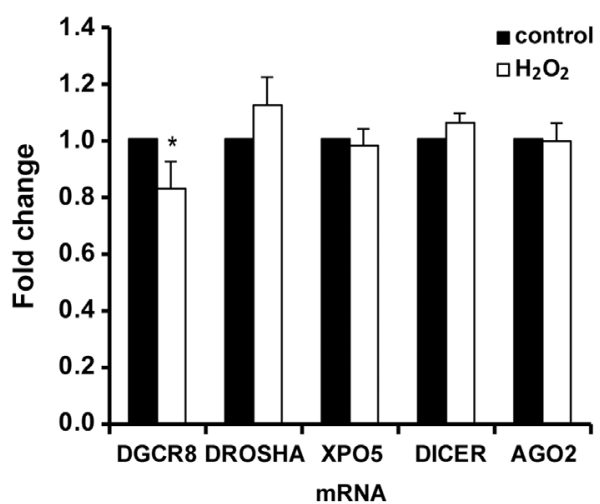

E

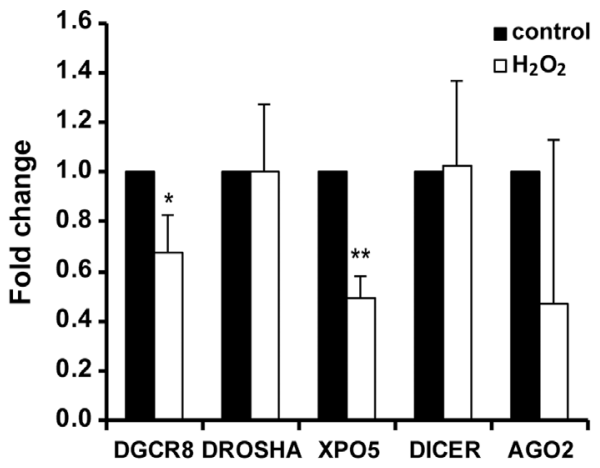

B

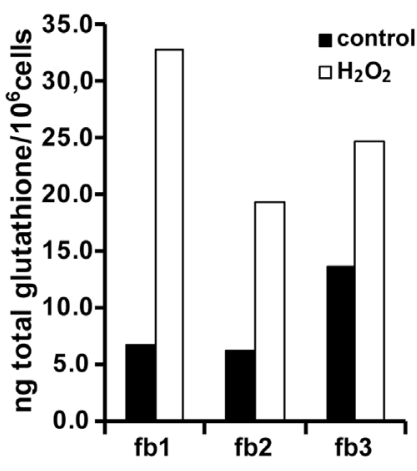

D

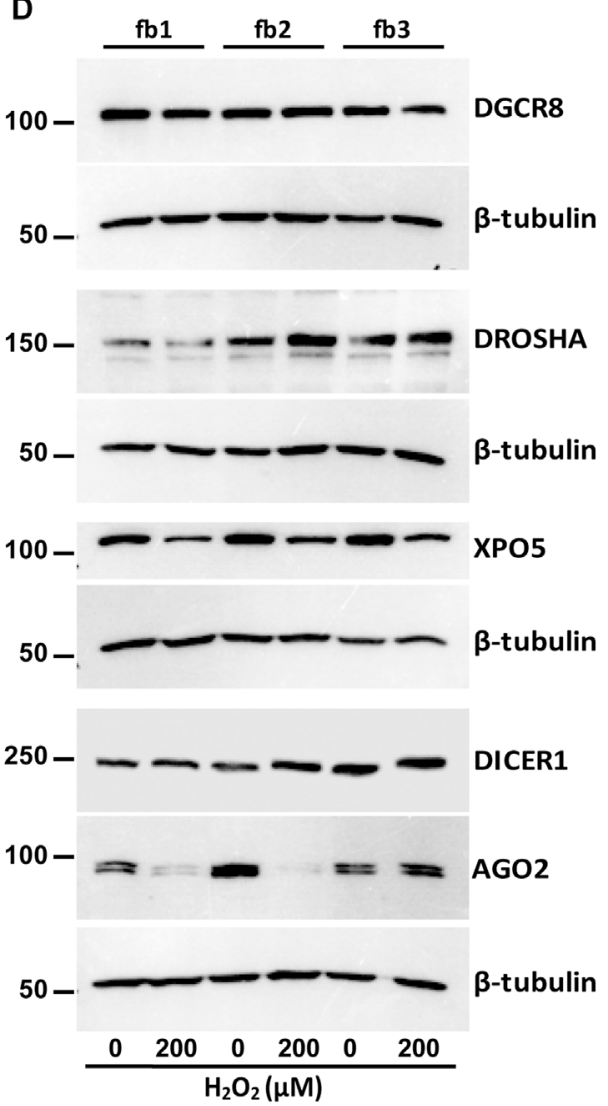

Fig. 5. Effects of oxidative stress on expression levels of miRNA biogenesis genes in dermal fibroblasts.

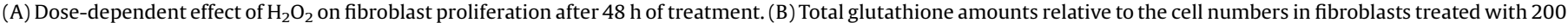

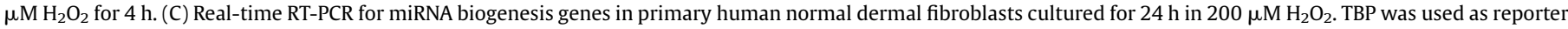

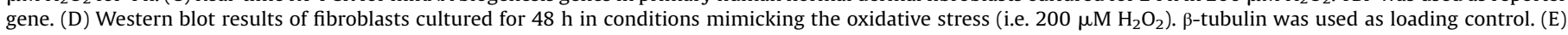
Densitometric analysis of western blot data on miRNA biogenesis proteins $\left({ }^{*} p<0.05 ;{ }^{* *} p<0.01 ;{ }^{* * *} p<0.001\right)$.

and XPO5, indicating that increased ROS activity may contribute to the observed reduction in genes responsible for miRNA biogenesis in this cell type. We can not exclude that alterations observed in miRNA biogenesis genes and modulation of their expression under hypoxic and oxidative stress conditions may also occur in other skin cell types.

The expression of pri-miRNAs for the same miRNAs analyzed for miRnome validation revealed that differences in expression levels between diabetic and non-diabetic samples are similar to those found for mature miRNAs. This result suggests that reduced miRNA levels are due to a more general transcriptional impairment that also affects pri-miRNA transcription.
In conclusion, our data confirm the complexity of molecular defects in diabetes, and that central mechanisms involved in the regulation of gene transcription are perturbed. They reinforce the concept that acting on the molecular machinery regulating gene expression can be pursued as a therapeutic option for diabetic complications.

\section{Declaration of Competing Interest}

The authors have no conflict of interest to declare. 


\section{Acknowledgments}

We wish to thank Lauretta Levati for experimental advice, Massimo Teson for dermal fibroblast isolation and Maurizio Inzillo for artwork. This study was supported by the Italian Ministry of Health (Ricerca Finalizzata RF-2009-1474128, to T.O; Ricerca Corrente to T.O. and to F.M; $5 \times 1000$ to F.M.).

\section{References}

[1] A.D. Deshpande, M. Harris-Hayes, M. Schootman, Epidemiology of diabetes and diabetes-related complications, Phys. Ther. 88 (2008) 1254-1264.

[2] L. Levy, J.A. Zeichner, Dermatologic manifestations of diabetes, J. Diabetes 4 (2012) 68-76

[3] H. Cho, M.R. Blatchley, E.J. Duh, S. Gerecht, Acellular and cellular approaches to improve diabetic wound healing, Adv. Drug Deliv. Rev. 18 (2018) 30193-30195 pii: S0169-409X [Epub ahead of print].

[4] V.P. Singh, A. Bali, N. Singh, A.S. Jaggi, Advanced glycation end products and diabetic complications, Korean J. Physiol. Pharmacol. 18 (2014) 1-14.

[5] C. Wu, X. Chen, J. Shu, C.-T. Lee, Whole-genome expression analyses of type 2 diabetes in human skin reveal altered immune function and burden of infection, Oncotarget 8 (2017) 34601-34609.

[6] D.M. Bermudez, B.J. Herdrich, J. Xu, R. Lind, D.P. Beason, M.E. Mitchell, L.J. Soslowsky, K.W. Liechty, Impaired biomechanical properties of diabetic skin implications in pathogenesis of diabetic wound complications, Am. J. Pathol. 178 (2011) 2215-2223.

[7] S. Horsburgh, N. Fullard, M. Roger, A. Degnan, S. Todryk, S. Przyborski, S O'Reilly, MicroRNAs in the skin: role in development, homoeostasis and regeneration, Clin. Sci. 131 (2017) 1923-1940.

[8] J. Moura, E. Børsheim, E. Carvalho, The role of microRNAs in diabetic complications-special emphasis on wound healing, Genes 5 (2014) 926-956.

[9] D. Ozdemir, M.W. Feinberg, MicroRNAs in diabetic wound healing: pathophysiology and therapeutic opportunities, Trends Cardiovasc. Med. 29 (2019) 131-137.

[10] R.C. Gentleman, V.J. Carey, D.M. Bates, B. Bolstad, M. Dettling, S. Dudoit, B. Ellis L. Gautier, Y. Ge, J. Gentry, K. Hornik, T. Hothorn, W. Huber, S. Iacus, R. Irizarry, F. Leisch, C. Li, M. Maechler, A.J. Rossini, G. Sawitzki, C. Smith, G. Smyth, L. Tierney, J.Y. Yang, J. Zhang, Bioconductor: open software development for computational biology and bioinformatics, Genome Biol. 5 (2004) R80.

[11] G.K. Smyth, Limma: linear models for microarray data, in: R. Gentleman, V. Carey, S. Dudoit, R. Irizarry, W. Huber (Eds.), Bioinformatics and Computationa Biology Solutions Using R and Bioconductor, Springer, New York, 2005, pp. 397-420.

[12] Y. Benjamini, Y Hochberg Controlling the false discovery rate: a practical and powerful approach to multiple testing, J. R. Stat. Soc. Ser. B 57 (1995) 289-300.

[13] I.S. Vlachos, N. Kostoulas, T. Vergoulis, G. Georgakilas, M. Reczko, M Maragkakis, M.D. Paraskevopoulou, K. Prionidis, T. Dalamagas, A.G. Hatzigeorgiou, DIANA miRPath v.2.0: investigating the combinatorial effect of microRNAs in pathways, Nucleic Acids Res. 40 (2012) W498-W504 Web Server issue.

[14] S. Ulisse, E. Baldini, S. Sorrenti, S. Barollo, L. Gnessi, A. Catania, M.R. Pellizzo, F. Nardi, C. Mian, E. De Antoni, M. D'Armiento, L. Frati, High expression of the urokinase plasminogen activator and its cognate receptor associates with advanced stages and reduced disease-free interval in papillary thyroid carcinoma, J. Clin. Endocrinol. Metab. 96 (2011) 504-508.

[15] S. Barrientos, O. Stojadinovic, M.S. Golinko, H. Brem, M. Tomic-Canic, Growth factors and cytokines in wound healing, Wound Repair Regen. 16 (2008) 585 601.

[16] X. Lim, R. Nusse, Wnt signaling in skin development, homeostasis, and disease, Cold Spring Harb, Perspect. Biol. Med. 5 (2013)a008029.

[17] W. Oi, C. Yang Z. Dai, D. Che, J.Feng, Y Mao, R. Cheng Z. Wang X. He, T. Zhou, X Gu, L. Yan, X. Yang, J.X. Ma, G. Gao, High levels of pigment epithelium-derived factor in diabetes impair wound healing through suppression of Wnt signaling, Diabetes 64 (2015) 1407-14019.

[18] G. Fitsialos, I. Bourget, S. Augier, A. Ginouvès, R. Rezzonico, T. Odorisio, F. Cianfarani, T. Virolle, J. Pouysségur, G. Meneguzzi, E. Berra, G. Ponzio, R. Buscà, HIF1 transcription factor regulates laminin-332 expression and keratinocyte migration, J. Cell. Sci. 121 (2008) 2992-3001.

[19] S.A. Eming, B. Brachvogel, T. Odorisio, M. Koch, Regulation of angiogenesis: wound healing as a model, Progr. Histochem. Cytochem. 42 (2007) 115-170.

[20] S. Barrientos, H. Brem, O. Stojadinovic, M. Tomic-Canic, Clinical application of growth factors and cytokines in wound healing, Wound Repair Regen. 22 (2014) 569-578.

[21] D. Ciarlillo, C. Celeste, P. Carmeliet, D. Boerboom, C. Theoret, A hypoxia response element in the Vegfa promoter is required for basal Vegfa expression in skin and for optimal granulation tissue formation during wound healing in mice, PLoS One 12 (2017)e0180586.

[22] M. Ha, V.N. Kim, Regulation of microRNA biogenesis, Nat. Rev. Mol. Cell Biol.15 (2014) 509-524.
[23] M. Kalani, K. Brismar, B. Fagrell, J. Ostergren, G. Jörneskog, Transcutaneous oxygen tension and toe blood pressure as predictors for outcome of diabetic foot ulcers, Diabetes Care 22 (1999) 147-151.

[24] T.A. Moretti Andrade, D. Santos Masson-Meyers, G. Ferreira Caetano, V.A. Terra, P. Payão Ovidio, A. Afonso Jordão-Júnior, M.A. Cipriani Frade, Skin changes in streptozotocin-induced diabetic rats, Biochem. Biophys. Res. Commun. 490 (2017) 1154-1161.

[25] D. Sigaudo-Roussel, C. Demiot, B. Fromy, A. Koïtka, G. Lefthériotis, P. Abraham, J.L. Saumet, Early endothelial dysfunction severely impairs skin blood flow response to local pressure application in streptozotocin-induced diabetic mice, Diabetes 53 (2004) 1564-1569.

[26] R. Rupaimoole, S.Y. Wu, S. Pradeep, C. Ivan, C.V. Pecot, K.M. Gharpure, A.S. Nagaraja, G.N. Armaiz-Pena, M. McGuire, B. Zand, H.J. Dalton, J. Filant, J. Bottsford Miller, C. Lu, N.C. Sadaoui, L.S. Mangala, M. Taylor, T. van den Beucken, E. Koch, C. Rodriguez-Aguayo, L. Huang, M. Bar-Eli, B.G. Wouters, M. Radovich, M. Ivan, G.A. Calin, W. Zhang, G. Lopez-Berestein, A.K. Sood, Hypoxia mediated downregulation of miRNA biogenesis promotes tumor progression, Nat. Commun. 5 (2014) 5202.

[27] T. van den Beucken, E. Koch, K. Chu, R. Rupaimoole, P. Prickaerts, M. Adriaens, J. M. Voncken, A.L. Harris, F.M. Buffa, S. Haider, M.H. Starmans, C.Q. Yao, M. Ivan, C. Ivan, C.V. Pecot, P.C. Boutros, A.K. Sood, M. Koritzinsky, B.G. Wouters, Hypoxia promotes stem cell phenotypes and poor prognosis through epigenetic regulation of DICER, Nat. Commun. 5 (2014) 5203.

[28] H. Thangarajah, D. Yao, E.I. Chang, Y. Shi, L. Jazayeri, I.N. Vial, R.D. Galiano, X.L. Du, R. Grogan, M.G. Galvez, M. Januszyk, M. Brownlee, G.C. Gurtner, The molecular basis for impaired hypoxia-induced VEGF expression in diabetic tissues, Proc. Natl. Acad. Sci. U.S.A. 106 (2009) 13505-13510.

[29] A. El-Osta, D. Brasacchio, D. Yao, A. Pocai, P.L. Jones, R.G. Roeder, M.E. Cooper, M. Brownlee, Transient high glucose causes persistent epigenetic changes and altered gene expression during subsequent normoglycemia, J. Exp. Med. 205 (2008) 2409-2417.

[30] F. Prattichizzo, V. De Nigris, R. Spiga, E. Mancuso, L. La Sala, R. Antonicelli, R. Testa, A.D. Procopio, F. Olivieri, A. Ceriello, Inflammageing and metaflammation: The yin and yang of type 2 diabetes, Ageing Res. Rev. 41 (2018) 1-17.

[31] J. Okano, H. Kojima, M. Katagi, T. Nakagawa, Y. Nakae, T. Terashima, T Kurakane, M. Kubota, H. Maegawa, J. Udagawa, Hyperglycemia induces skin barrier dysfunctions with impairment of epidermal integrity in non-wounded skin of type 1 diabetic mice, PLoS One 11 (2016)e0166215.

[32] V.M. Pham, S. Matsumura, T. Katano, N. Funatsu, S. Ito, Diabetic neuropathy research: from mouse models to targets for treatment, Neural Regen. Res. 14 (2019) 1870-1879.

[33] P. Algenstaedt, C. Schaefer, T. Biermann, A. Hamann, B. Schwarzloh, H. Greten, W. Rüther, N. Hansen-Algenstaedt, Microvascular alterations in diabetic mice correlate with level of hyperglycemia, Diabetes 52 (2003) 542-549.

[34] A. Ibuki, T. Akase, T. Nagase, T. Minematsu, G. Nakagami, M. Horii, H. Sagara, T. Komeda, M. Kobayashi, T. Shimada, M. Aburada, K. Yoshimura, J. Sugama, H. Sanada, Skin fragility in obese diabetic mice: possible involvement of elevated oxidative stress and upregulation of matrix metalloproteinases, Exp. Dermatol. 21 (2012) 178-183.

[35] H. Thangarajah, I.N. Vial, R.H. Grogan, D. Yao, Y. Shi, M. Januszyk, R.D. Galiano, E.I. Chang, M.G. Galvez, J.P. Glotzbach, V.W. Wong, M. Brownlee, G.C. Gurtner, HIF-1alpha dysfunction in diabetes, Cell Cycle 9 (2010) 75-79.

[36] S.-B. Catrina, X. Zheng, Disturbed hypoxic responses as a pathogenic mechanism of diabetic foot ulcers, Diabetes Metab. Res. Rev. 32 (2016) 179-185.

[37] I. Petit, D. Jin, S. Rafii, The SDF-1-CXCR4 signaling pathway: a molecular hub modulating neo-angiogenesis, Trends Immunol. 28 (2007) 299-307.

[38] S. Greco, P. Fasanaro, S. Castelvecchio, Y. D’Alessandra, D. Arcelli, M. Di Donato, A. Malavazos, M.C. Capogrossi, L. Menicanti, F. Martelli, MicroRNA dysregulation in diabetic ischemic heart failure patients, Diabetes 61 (2012) $1633-1641$

[39] A. Bugyei-Twum, A. Advani, S.L. Advani, Y. Zhang, K. Thai, D.J. Kelly, K.A. Connelly, High glucose induces Smad activation via the transcriptional coregulator p300 and contributes to cardiac fibrosis and hypertrophy, Cardiovasc. Diabetol. 13 (2014) 89.

[40] I. Russo, N.G. Frangogiannis, Diabetes-associated cardiac fibrosis: Cellular effectors, molecular mechanisms and therapeutic opportunities, J. Mol. Cell. Cardiol. 90 (2016) 84-93.

[41] Y.C. Qiao, Y.L. Chen, Y.H. Pan, W. Ling, F. Tian, X.X. Zhang, H.L. Zhao, Changes in transforming growth factor beta 1 in patients with type 2 diabetes and diabetic nephropathy. A PRISMA-compliant systematic review and meta-analysis, Medicine 96 (2017) 15 e6583.

[42] I. Ackers, R. Malgor, Interrelationship of canonical and non-canonical Wnt signalling pathways in chronic metabolic diseases, Diab. Vasc. Dis. Res. 15 (2018) 3-13.

[43] E.L. Feldman, K.A. Nave, T.S. Jensen, D.L.H. Bennett, New horizons in diabetic neuropathy: mechanisms, bioenergetics, and pain, Neuron. 93 (2017) 12961313. 